diagnosed with EILO; i.e. they had both conditions. Treatment for EILO resolved breathing problems in the majority.

Conclusions EILO is common in athletes, but too often referred to as 'mysterious breathing problems'. Asthma does not rule out EILO as comorbidities are common. Apparent persistence of respiratory symptoms must not lead to indiscriminate escalation of asthma treatment, as symptoms may be due to undiagnosed and treatable laryngeal obstruction.

\section{PREVENTING INJURY TO THE FETUS: USING 3- DIMENSIONAL POWER FLOW DOPPLER ULTRASONOGRAPHY TO ANALYZE PLACENTAL BLOOD FLOW DURING RESISTANCE TRAINING IN PREGNANT ATHLETES TO GUARD AGAINST FETAL HYPOPERFUSION}

\begin{abstract}
${ }^{1,4}$ Sara Gould, ${ }^{2}$ Chase Cawyer, ${ }^{3,4}$ Louis Dell'Italia, ${ }^{2}$ Lorie Harper, ${ }^{4,5}$ Marcas Bamman. ${ }^{1}$ University of Alabama at Birmingham, Department of Orthopedics, Division of Sports Medicine, AL, USA; ${ }^{2}$ University of Alabama at Birmingham, Department of Obstetrics and Gynecology, Division of Maternal-Fetal Medicine, AL, USA; ${ }^{3}$ University of Alabama at Birmingham, Department of Medicine, Division of Cardiovascular Disease, AL, USA; ${ }^{4}$ Birmingham Veteran Affairs Medical Center, AL, USA; ${ }^{5}$ University of Alabama at Birmingham, Department of Cell, Developmental, and Integrative Biology, AL, USA
\end{abstract}

\subsection{6/bjsports-2021-IOC.271}

Background Peak fertility and athletic performance coincide, placing a subset of women into competition with sparse guidance. The Valsalva maneuver has been hypothesized to increase maternal blood pressure and intra-abdominal pressure, resulting in decreased blood flow to the fetus during resistance training.

Objective We present a technique employing three dimensional (3D) power Doppler ultrasound analysis to evaluate placental blood flow during resistance exercise. This technique may be used to prevent fetal injury by guiding training parameters.

Design This is a proof of principal study describing the technique and documenting its use to determine placental blood flow.

Setting This research was conducted in an academic clinical enter. Participants varied from an actively exercising, former collegiate athlete, to participants who performed no scheduled physical activity.

Patients (or Participants) Four normal weight women less than 35 years of age with uncomplicated pregnancies were included.

Interventions (or Assessment of Risk Factors) One repetition maximum (1RM) via modified chest press was determined. Ultrasonography with power Doppler and 3D volume measurements were then performed on the visualized portions of the placenta. The vascular flow index (VFI) was then calculated for each phase.

Main Outcome Measurements VFI was measured during lifting and at rest. The paired t-test was used for statistical analysis. $\mathrm{Z}$ scores from previously established normative data (Noguchi, et al 2009) provided benchmarks.

Results VFI was not significantly different between lift and rest phase for any of our participants. All measurements were within a standard deviation of previously established normative data. There were no incidences of fetal bradycardia.

Conclusions 3D power flow Doppler imaging can guide resistance training during pregnancy to prevent fetal injury due to hypoperfusion. Resistance training up to an RM1 of 50lbs did not result in a significant reduction in placental blood flow from resting state in the study population.

\section{ABSTRACT WITHDRAWN}

\section{EFFECTS OF SALBUTAMOL PRIOR TO DIESEL EXHAUST EXPOSURE DOES NOT AFFECT DYSPNEA DESPITE REDUCING THE WORK OF BREATHING IN INDIVIDUALS WITH EXERCISE-INDUCED BRONCHOCONSTRICTION}

${ }^{1}$ Sarah Koch, ${ }^{2}$ Joseph Welch, ${ }^{1}$ Andrew Ramsook, ${ }^{1}$ Christopher Carlsten, ${ }^{1} J o r d a n$ A Guenette, 'Michael Stephen Koehle. 'University of British Columbia, Vancouver, Canada; ${ }^{2}$ University of Florida, Gainesville, USA

\subsection{6/bjsports-2021-IOC.272}

Background Individuals with exercise-induced bronchoconstriction (EIB) use inhaled $\beta_{2}$-agonists prior to exercise to prevent respiratory symptoms. The resulting bronchodilation could increase the dose of inhaled pollutants and worsen dyspnea when exercise is performed in air pollution.

Objective To assess the effects of salbutamol, a $\beta_{2}$-agonist, and diesel exhaust (DE) exposure during exercise on dyspnea and the metabolic cost of breathing in individuals with EIB.

Design Double-blind, randomised, repeated measures.

Setting Controlled human exposure study.

Participants Nineteen participants $(9$ women) with EIB (age 22-33 years).

Intervention Participants completed four exposure visits. After the inhalation of either $400 \mu \mathrm{g}$ of salbutamol or placebo, participants sat in the exposure chamber for $60 \mathrm{~min}$, breathing either filtered air (FA) or DE $\left(\mathrm{PM}_{2.5}=300 \mu \mathrm{g} / \mathrm{m}^{3}\right)$. Following the rest period, participants cycled for $30 \mathrm{~min}$ at $50 \%$ of peak work rate while breathing FA or DE.

Main Outcome Measurements Dyspnea was rated using the 0 10 category-ratio Borg scale at baseline, $7.5 \mathrm{~min}$ and $27.5 \mathrm{~min}$ into the cycling bout. Transpulmonary pressure was calculated as the difference between esophageal and mouth pressure. The work of breathing (WOB) was then determined by integrating ensemble averaged transpulmonary pressure-tidal volume loops.

Results Dyspnea increased significantly $(\mathrm{p}<0.01)$ from $0 \pm 1$ at baseline to $2 \pm 1$ at $7.5 \mathrm{~min}$ and $3 \pm 2$ at $27.5 \mathrm{~min}$. Neither DE $(p=0.71)$ nor salbutamol $(p=0.45)$ affected dyspnea WOB increased with exercise duration, starting at $8.3 \pm$ $6.8 \mathrm{~J} / \mathrm{min}$ at baseline to $95.5 \pm 35.9 \mathrm{~J} / \mathrm{min}(\mathrm{p}<0.01)$ at $7.5 \mathrm{~min}$ and $105.0 \pm 47.5 \mathrm{~J} / \mathrm{min}(\mathrm{p}<0.01)$ at $27.5 \mathrm{~min}$. Exposure to DE did not affect WOB $(\mathrm{p}=0.49)$; however, salbutamol reduced WOB significantly $(p=0.049)$. At $7.5 \mathrm{~min}$ in the salbutamol trial, WOB was reduced by $10.8 \pm 2.9 \mathrm{~J} /$ min compared to placebo $(p=0.048)$. At $27.5 \mathrm{~min}$, WOB was reduced by $3.9 \pm 1.3 \mathrm{~J} / \mathrm{min}$ after salbutamol compared to placebo although this did not reach statistical significance $(\mathrm{p}=0.12)$.

Conclusions The use of salbutamol prior to exercise in DE does not appear to affect dyspnea despite reducing the WOB in individuals with EIB. 